\title{
Intellectual aspects of cognitive performance in children after treatment for medulloblastoma and astrocytoma
}

\author{
Izabel Hazin', Georges Dellatolas², Danielle Garcia ${ }^{1}$, Francisco Pedrosa ${ }^{3}$, and Arli Pedrosa ${ }^{3}$ \\ 1- Universidade Federal do Rio Grande do Norte, Natal, RN, Brazil \\ 2- Université Paris Descartes, Paris, IDF, France \\ 3- Instituto Materno Infantil Professor Fernando Figueira, Recife, PE, Brazil
}

\begin{abstract}
The present study investigated the relationship between posterior fossa tumors (astrocytoma and medulloblastoma) and their respective treatments and cognitive performance in Brazilian children by measuring Intelligence Quotient (IQ). Twenty children were enrolled in the study, of whom 13 were diagnosed with astrocytoma (average age at evaluation, 10.2 years; eight girls and five boys) and seven were diagnosed with medulloblastoma (average age at evaluation, 9.2 years; five girls and two boys). The first subgroup underwent exclusively tumor resection surgery, and the second subgroup underwent surgery, chemotherapy (Vincristine, Cisplatine, and Carmustine), and radiotherapy (total dose, $54 \mathrm{~Gy}$ ). The inclusion criteria for the clinical group were normal social and emotional behavior before the diagnosis and motor and visual performance not excessively deteriorated after surgery. All participants were submitted to the Wechsler Intelligence Scale for Children - third version, and their scores were related to standard norms established for Brazilian children and compared with regard to the diagnosis subgroups and treatment modalities. Statistically significant differences were identified between the two diagnosis subgroups in Performance IQ and Processing Speed scores. The medulloblastoma subgroup presented poorer performance in all domains compared with the astrocytoma subgroup. Time interval between diagnosis and neuropsychological evaluation also had a significant effect on Processing Speed and Freedom from Distractibility in the medulloblastoma subgroup. These results provide empirical evidence of a possible significant effect of radiotherapy exposure on processing speed and global intellectual capacity. Keywords: posterior fossa tumor, radiotherapy, intelligence evaluation.
\end{abstract}

Received 6 October 2010; received in revised form 7 December 2010; accepted 11 December 2010. Available on line 28 December 2010

\section{Introduction}

Cancer among children represented nearly $2.5 \%$ of the newly cases of neoplasias estimated in Brazil in 2010. Despite this apparently small figure, it represents more than 9000 yearly diagnosed cases of neoplasies among children and adolescents aged 18 years or less. About $8-15 \%$ of all diagnosed pediatric neoplasies are central nervous system (CNS) tumors, with this condition being the most frequently diagnosed case of solid tumors within this age group, especially among male children under 15 years of age. The highest incidence occurs at 10 years of age (Instituto Nacional de Câncer, 2009).

Izabel Hazin, Danielle Garcia, Universidade Federal do Rio Grande do Norte, Natal, RN, Brazil. Georges Dellatollas, Université Paris Descartes, Paris, France. Francisco Pedrosa, Arli Pedrosa, Instituto Materno Infantil Professor Fernando Figueira/ Centro de Hematologia e Oncologia Pediátrica, Recife, PE, Brazil. Correspondence regarding this article should be directed to: Izabel Hazin, Universidade Federal do Rio Grande do Norte, CCHLA, Av. Senador Salgado Filho, s/n, Lagoa Nova, Natal, RN, Brazil, CEP 59078-000. E-mail: izabel.hazin@gmail.com
CNS tumors in children have peculiar characteristics. Whereas supratentorial lesions are more frequent in adults, infratentorial lesions, also known as posterior fossa tumors, are more common in children. This type of lesion occurs in the region under the tentorium, which is a dura mater membrane that covers the cerebellum and sustains the occipital lobes of the brain (Mabbott, Penkman, Witol, Strother, \& Bouffet, 2008).

The cerebellum has been associated with higherorder cognitive function, particularly cognitive function related to language, planning, emotion, and the ability to sequentially organize symbolic activity. Recent studies have demonstrated the existence of connections between the cerebellum and dorsolateral prefrontal cortex related to executive function, operational memory, and attention (Bugalho, Correa, \& Viana-Baptista, 2006). Cognitive alterations caused by cerebellar lesions led to the proposition of two associated syndromes: Posterior Fossa Syndrome and Cerebellar Cognitive Affective Syndrome. Both of these syndromes present a relatively regular set of cognitive and behavioral alterations associated with focal lesions of the cerebellum. Posterior Fossa Syndrome is initially characterized by the presence 
of transient cerebellar mutism, followed by dysarthria. It is an iatrogenic complication resulting from surgical removal of posterior fossa tumors that affect the midline or require incisions of the cerebellar vermis. Posterior Fossa Syndrome also presents a well-defined set of associated cognitive and behavioral deficits, including verbal fluency alterations, visuospatial function alterations, executive deficits, visual memory deficits, and affective alterations (Bugalho et al., 2006).

Greater knowledge of the pathophysiology of cancer associated with highly refined therapies has increased the survival rates of children diagnosed with CNS tumors. However, the combination of different modalities of treatment that promote the efficacy of therapies can also elicit adverse physical, cognitive, emotional, and social conditions, which are attributed to the aggressiveness of the therapies and the neoplasia itself (Nathan et al., 2007).

Surgery is the quickest and most effective method for tumor reduction and is often the first-line therapeutic intervention for CNS tumors. The degree of tumor resection is a factor that has great prognostic value. The existence of residual lesions often determines the necessity for adjuvant therapeutic interventions (Palmer, 2009). The extent of tumor resection is sometimes used for staging individual patients. Primary tumors not totally resected are commonly considered high risk and determine that additional treatment for the child is necessary. Benign tumors, such as pilocytic astrocytomas, usually require only resection surgery, although chemotherapy has been included in some protocols to reduce the risk of relapse. Nevertheless, for malignant tumors, which generally present residual tumor tissue, relapse, or metastasis, surgical treatment is supplemented by chemotherapy or radiotherapy.

In a recent literature review, Palmer (2009) referred to the paucity of studies that have investigated the impact of combined treatment (i.e., surgery, radiotherapy, and chemotherapy for posterior fossa tumors, notably medulloblastomas) on cognitive function. This treatment is considered a high-risk approach, despite the facts that it is usually considered to have favorable outcomes, is used for children under 3 years of age, and is included in European protocols.

This exploratory study investigated the impact of posterior fossa tumors (medulloblastomas and astrocytomas) and their corresponding treatments on the intellectual capacity of children from the Northeastern geopolitical region of Brazil. The time that elapsed between diagnosis and neuropsychological evaluation was also considered in the evaluation of these groups. The children were treated at the Centro de Hematologia e Oncologia Pediátrica (CEHOPE, Centre of Haematology and Paediatric Oncology) in Recife, the capital city of the geopolitical state of Pernambuco.
Notably, the Northeastern region, together with the Northern region, has the worst indices of development in Brazil. The population treated at CEHOPE belongs to underprivileged social groups, and approximately $85 \%$ come from the countryside of Pernambuco, where severe socioeconomic difficulties have led to malnutrition, one of the highest child mortality rates in the country (41.2 per thousand children born alive), and poor health and education conditions.

\section{Methods}

\section{Subjects}

Twenty children were enrolled in the study. The astrocytoma (AS) group comprised 13 children (eight girls and five boys), of whom five had vermal tumors and eight had hemispheric tumors. The average age of the AS group was 10.2 years (range 7-14 years) at evaluation and 9.2 years (range 4-13 years) at surgery. Eight children $(61.5 \%)$ in the AS group underwent treatment of hydrocephalus by ventriculoperitoneal shunt. The medulloblastoma (ME) group comprised seven participants (five girls and two boys), of whom three had vermal tumors and four had hemispheric tumors. The average age of the ME group was 10.2 years (range 8-16 years) at evaluation and 6.5 years (range 2-11 years) at surgery. Four children (57\%) in the ME group underwent treatment of hydrocephalus by ventriculoperitoneal shunt.

The children diagnosed with pilocytic astrocytoma (low-grade glioma) were treated with tumor resection surgery alone. The children diagnosed with medulloblastoma (all of whom were high risk, with evidence of metastatic disease or residual tumors after surgery $>1.5 \mathrm{~cm}^{3}$ ), in addition to surgery, underwent radiotherapy (total dose, $54 \mathrm{~Gy} ; 36 \mathrm{~Gy}$ for craniospinal +18 Gy for tumor bed) and chemotherapy (Vincristine, Cisplatine, and Carmustine).

The inclusion criteria for the clinical groups were normal social and emotional behavior before the diagnosis and motor and visual performance not excessively deteriorated after surgery. Table 1 summarizes the main characteristics of the clinical groups.

The sample distribution of the participants was not controlled by gender because this variable was not equally distributed in the reference population of patients in the medical service from where participants came.

The present research complied with all regulations of Resolution 196 of the Conselho Nacional de Saúde do Brasil (National Health Council of Brazil), which determines the norms and guidelines for research involving human subjects. The research was approved by the ethics and research committee of the Instituto Materno Infantil Prof. Fernando Figueira (Recife, Brazil), which is the medical and research institution with which CEHOPE is affiliated. 
Table 1. General profile of the children in the clinical groups included in the study (number of children in each condition with corresponding percentage of clinical group).

\begin{tabular}{lcc}
\hline \multirow{2}{*}{ Parameter } & \multicolumn{2}{c}{ Clinical Group } \\
\cline { 2 - 3 } Number of subjetcs & Astrocytoma & Medulloblastoma \\
Age at diagnosis & $13(65.0 \%)$ & $7(35.0 \%)$ \\
Male & $4-13$ years (Mean: 9.2 years) & $2-12$ years (Mean: 6.5 years) \\
Female & $5(38.5 \%)$ & $2(28.6 \%)$ \\
Time since diagnosis $\leq 3$ years & $8(61.5 \%)$ & $4(52.9 \%)$ \\
Patients under 6 years of age at diagnosis & $9(69.2 \%)$ & $5(71.4 \%)$ \\
\hline
\end{tabular}

\section{Neuropsychological evaluation: instrument}

For the evaluation of intellectual capacity, the present study used the Wechsler Intelligence Scale for Children third version (WISCIII; Figueiredo, 2002). The use of such an instrument did not have the unique goal of determining Intelligence Quotient (IQ) scores that could justify the lower global intellectual achievement of children exposed to cancer treatment. This instrument was used to achieve a better understanding of the interdependency of psychological functions for this specific subgroup of children. The choice of such a scale is justified by the concept of intelligence proposed by David Wechsler, in which intelligence is more an effect than a cause and can be described as a set of intellectual and nonintellectual components that enable the individual to act intentionally, think, and deal with the environment.

The WISCIII is composed of 13 subtests that, when grouped in a specific manner, provide three IQ scores (full scale IQ [FSIQ], verbal IQ [VIQ], and performance IQ [PIQ]) and four factor indices (verbal comprehension index [VCI], perceptual organization index [POI], Freedom from Distractibility index [FDI], and processing speed index [PSI]). The four factor indices reflect specific aspects of cognitive function. According to Figueiredo (2002), the VCI and POI are the purest dimensions of the verbal and nonverbal domains, respectively. The FDI, which refers to the verbal domain, evaluates concentration and immediate memory and is strongly related to mathematical ability. Finally, the PSI reflects psychomotor and mental speed.

\section{Procedures}

The children who participated in this study were submitted to the WISCIII in two sessions, 60 min each, with a time delay of 2 weeks between each session. These sessions were conducted in an appropriate room at CEHOPE. All of the children's families were informed about the conditions of participation, which were read to them as they were written in the regular informed consent (Termo de Consentimento Livre e
Esclarecido), which is regulated by Brazilian norms concerning research with human subjects.

\section{Data analysis}

Data were analyzed using descriptive and inferential tools, with nonparametric tests in the latter case, considering the small number of participants and the non-guaranteed normal distribution of the data in the population of origin. The following two approaches for analysis were used: verification of contrast between scores from WISCIII (IQs and factor indices) related to (i) the instances of the independent variables considered for the global set of participants in the present study and (ii) the instances of the independent variables considered for the subgroups of children with astrocytoma (AS) vs. medulloblastoma (ME).

\section{Results}

The analysis of WISCIII performance in the two groups indicated better achievement in the AS group for all dimensions compared with the ME group. In the AS group, the mean FSIQ score was 95.5 (standard deviation $[S D]=17.8$ ), the mean VIQ score was 98.8 $(S D=19.6)$, and the mean PIQ score was $92.9(S D=$ 16.1). In the ME group, the FSIQ score was 85.7 ( $S D$ $=16.5)$, the mean VIQ score was $97.7(S D=16.4)$, and the mean PIQ score was $75.8(S D=16.1)$. A significant difference (> 15 points) was identified between VIQ and PIQ scores for $10(50 \%)$ of the 20 patients. For all of these patients (four from the AS group and six from the ME group), VIQ scores were higher than PIQ scores.

Data from the groups' performance for each factor index showed the superiority of the AS group over the ME group, with the exception of the VCI, for which the ME group had slightly better performance. The global results for both groups were characterized by assessing VCI, POI, FDI, and PSI, with the following values, respectively: $99.5(S D=16.8), 88.1(S D=16.3), 92.9$ $(S D=18.6)$, and $86.3(S D=16.3)$. The mean performance 
of the AS group for each factor index was, respectively, $99.3(S D=17.9), 92.9(S D=15.3), 94.4(S D=21)$, and $93.3(S D=14)$. The mean performance of the ME group for each factor index was, respectively, 99.9 (SD $=16.1), 79.1(S D=15.1), 90(S D=14.3)$, and $73.1(S D$ $=11.8$ ). Importantly, a significant difference was found between the two groups for PIQ score $(p=.02$, MannWhitney $U=18)$ and PSI score $(p=.01$, Mann-Whitney $U=13)$. The analysis of the WISCIII subtests indicated that for the verbal domain, the lowest mean scores for both groups were on the Information subtest (which assesses formal knowledge acquired mainly in school) and Arithmetic subtest (which assesses mathematical reasoning and working memory).

The analysis of achievement in both groups for the subtests of the performance domain revealed important differences between groups. The only subtest for which the AS group had a mean performance score lower than expected was Picture Arrangement (weighted mean score =7.7). However, the ME group had lower than expected mean scores for all subtests, particularly the Blocks and Picture Arrangement subtests, which assess nonverbal reasoning and visuospatial organization, and the Symbols subtest, which assesses mental and psychomotor speed.

\section{Verification of contrast between WISCIII scores (IQ} scores and factor indices) according to treatment type

Of the participants in the present study, 13 (AS group) were diagnosed with astrocytoma of the posterior fossa and underwent a treatment protocol that consisted exclusively of neurosurgery for tumor resection, and seven (ME group) were diagnosed with high-risk posterior fossa medulloblastoma and underwent a treatment protocol consisting of neurosurgery for tumor resection combined with chemotherapy (Vincristine, Cisplatine, and Carmustine) and radiotherapy (total dose, 54 Gy). As discussed previously, the ME group presented poorer performance in all domains compared with the AS group.

The difference between groups was significant for PIQ score ( $p=.02$, Mann-Whitney $U=18)$, which is an intelligence dimension related to nonverbal ability. With regard to the analysis of factor indices, a highly significant difference was found between groups for PSI $(p=.01$, MannWhitney $U=13$ ). These results indicate lower mental and psychomotor speed in the ME group, which contributed to the low PIQ scores. These findings are consistent with the role ascribed to information processing speed for intelligence evaluation, suggesting that radiotherapy may impact processing speed (Palmer, 2009).

Verification of contrast between WISCIII scores (IQ scores and factor indices) according to the time elapsed between diagnosis and neuropsychological evaluation

The variable representing the time elapsed between diagnosis and neuropsychological evaluation did not show a significant difference in WISCIII scores in the
AS group (Mann-Whitney $U$ test, $p>.05$ ) but did show a significant difference in the ME group (Mann-Whitney $U$ test, $p<.01$ ), indicating a progressive cognitive decline in patients subjected to cranial radiotherapy. The mean IQ scores for patients in the ME group with an interval between diagnosis and neuropsychological evaluation of 3 years or less were FSIQ $=96(S D=87)$, VIQ $=$ $107.5(S D=6.6)$, and PIQ $=85.2(S D=13)$. The results for the same IQ scores in the subgroups with an interval between diagnosis and neuropsychological evaluation greater than 3 years were FSIQ $=72(S D=14.7), \mathrm{VIQ}=$ 84.6 $(S D=17.2)$, and PIQ $=63.3(S D=10.5)$.

Statistically significant results were found in the analysis of scores from the ME group for FDI (MannWhitney $U$ test, $p=.01$ ) and PSI (Mann-Whitney $U$ test, $p=.01$ ) when the variable representing the time elapsed between diagnosis and neuropsychological evaluation was considered. The scores for FDI and PSI in the subgroup with an interval between diagnosis and neuropsychological evaluation of 3 years or less were FDI $=98.7(S D=8.3)$ and PSI $=79.7(S D=11.7)$. The scores for the same indices in the second subgroup, with the time elapsed between diagnosis and neuropsychological evaluation greater than 3 years, were FDI $=78.3(S D=$ $12.5)$ and PSI $=64.3(S D=3.5)($ Figure 1$)$.

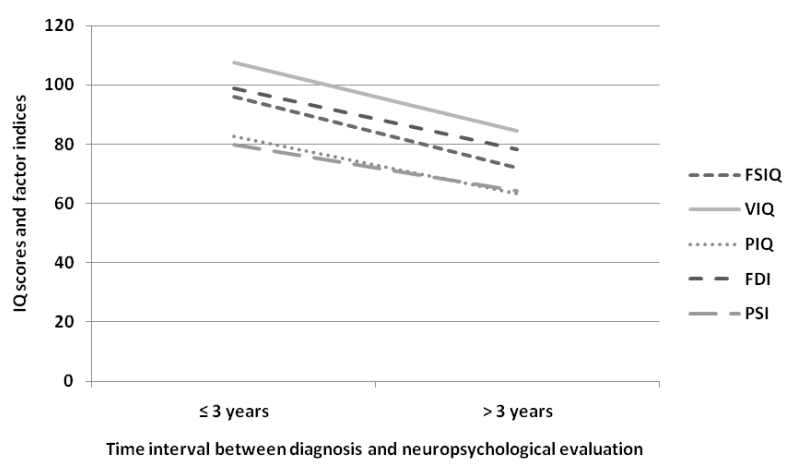

Figure 1. Comparison between IQ scores and factor indices in patients in the ME group according to the time interval between diagnosis and neuropsychological evaluation.

\section{Discussion}

The aim of the present study was to investigate the impact of posterior fossa tumors and their treatments on the intellectual capacity of Brazilian children diagnosed with medulloblastoma and astrocytoma. The analysis of the performance of these groups revealed significant differences in WISCIII scores in the domains of full scale, verbal, and performance intelligence and in more detailed levels of capacity, assessed by the factor indices scores.

The comparison of performance in the group diagnosed with astrocytoma and submitted exclusively to tumor resection surgery and the group diagnosed 
with high-risk medulloblastoma and subjected to tumor resection surgery combined with chemotherapy and cranial radiotherapy revealed that the former group had scores in all dimensions that are consistent with the expected results for the reference population of the same age range and normative WISCIII data for the Brazilian population. The subgroup of patients with medulloblastoma presented significant deficits in the PIQ domain, reflected in significant differences in the scores between the verbal domain and performance domain for more than $90 \%$ of the patients. The FSIQ score, therefore, cannot be considered sufficiently representative of global intellectual capacity. Such findings are consistent with studies that demonstrated the absence of a significant impact on VIQ scores in patients with different posterior fossa tumors (Von Hoff, Kieffer, Habrand, Kalifa, Dellatolas, \& Grill, 2008). However, a significant impact on PIQ scores was found in the medulloblastoma and astrocytoma subgroups (Palmer, 2009). A possible association between radiotherapy and lower intellectual capacity was suggested previously, particularly in the domain of nonverbal ability (Vaquero, Gómez, Quintero, González-Rosa, \& Márquez, 2008; Palmer, Reddick, \& Gajjar, 2007).

In the analysis of mean scores for the factor indices that comprise the PIQ, the lowest scores in the subgroup of patients with medulloblastoma were obtained with PSI. The speed at which information is processed at mental and psychomotor levels is directly associated with white matter density. Alterations in this dimension, notably in terms of developmental failures, directly affect information processing speed, which significantly impacts global intellectual capacity (Mabbott et al., 2008). Research in developmental psychology indicates a strong link between processing speed and better working memory performance and the capacity for acquiring new knowledge and abilities, expressed through IQ scores (Palmer, 2009).

Evidence has shown an association between cranial radiotherapy and a reduction in white matter, which led Khong et al. to suggest that white matter volume could be used as a biological marker to verify the neurotoxicity of radiotherapy (Khong et al., 2006). The fact that children subjected to radiotherapy do not process information at the same speed as their peers leads to significant gaps that negatively affect the development of their abilities and acquisition of new knowledge (Mabbot et al., 2008).

Importantly, IQ values decreased with time only in children with medulloblastoma (i.e., a progressive impact of the disease and its treatment on intellectual performance was identified in this group). Participants with a time interval between diagnosis and neuropsychological evaluation greater than 3 years had lower scores on all domains of the WISCIII compared with the subgroup with an average time interval of 3 years or less. Similar findings were reported by Merchant et al. (Merchant, Conklin, Wu, Lustig, \& Xiong,
2009) in a prospective study of 78 children who had survived posterior fossa tumors. In this study, a progressive decline in global intellectual capacity was identified 5 years after radiotherapy. A study by Stargatt et al (2006). discussed the risks associated with cerebral tumors during childhood with regard to progressive damage of intellectual capacity that increases with time (Stargatt, Rosenfeld, Anderson, Hassall, Maixner, \& Ashley, 2006).

The present study was limited by the low number of participants, which was due to the rarity of the studied diseases and low rate of survival among patients in the studied region of Brazil. Additional longitudinal studies are necessary to produce complementary psychometric data.

\section{Acknowledgements}

This study was undertaken with the support of two Brazilian research funding agencies: Coordenação de Aperfeiçoamento de Pessoal de Nível Superior (postdoctoral grant awarded to the first author) and Conselho Nacional de Desenvolvimento Científico e Tecnológico.

\section{References}

Bugalho, P., Correa, B., \& Viana-Baptista, M. (2006). Papel do cerebelo nas funções cognitivas e comportamentais: bases científicas e modelos de estudo. Acta Medica Portuguesa, 19, 257-268.

Figueiredo, V.L.M. (2002). WISC III. In J.A. Cunha (Ed.), Psicodiagnóstico V (pp. 603-614). Porto Alegre: Artmed.

Instituto Nacional de Câncer (2009). Estimativa 2010: Incidência de câncer no Brasil. Retrieved from http://www.inca.gov.br/ estimativa/2010/index.asp?

Khong, P.L., Leung, L.H.T., Fung, A.S.M., Fong, D.Y.T., Qiu, D., Kwong, D.L.W.,... Chan, G.C.F. (2006). White matter anisotropy in post-treatment childhood cancer survivors: preliminary evidence of association with neurocognitive function. Journal of Clinical Oncology, 24, 884-890.

Mabbott, D.J., Penkman, L., Witol, A., Strother, D., \& Bouffet, E. (2008). Core neurocognitive functions in children treated for posterior fossa tumors. Neuropsychology, 22, 159-168.

Merchant, T.E., Conklin, H.M., Wu, S., Lustig, R.H., \& Xiong, X. (2009). Late effects of conformal radiation therapy for pediatric patients with low-grade glioma: prospective evaluation of cognitive, endocrine, and hearing deficits. Journal of Clinical Oncology, 27, 3691-3697.

Nathan P.C., Patel S.K., Dilley, K., Goldsby R., Harvey, J., Jacobsen, ... Armstrong, F.D. (2007). Guidelines for identification of, advocacy for, and intervention in neurocognitive problems in survivors of childhood cancer: a report from the Children's Oncology Group. Archives of Pediatric and Adolescent Medicine, 161, 798-806.

Palmer, S.L. (2009). Neurodevelopmental impact on children treated for medulloblastoma: a review and proposed conceptual model. Developmental Disabilities Research Reviews, 14, 203-210.

Palmer, S.L., Reddick, W.E., \& Gajjar, A. (2007). Understanding the cognitive impact on children who are treated for medulloblastoma. Journal of Pediatric Psychology, 32, 1040-1049.

Stargatt, R., Rosenfeld, J.V., Anderson, V., Hassall, T., Maixner, W., \& Ashley, D. (2006). Intelligence and adaptive function in children diagnosed with brain tumour during infancy. Journal of Neurooncology, 80, 295-303.

Vaquero, E., Gómez, C.M., Quintero, E.A., González-Rosa, J.J., \& Márquez, J. (2008). Differential prefrontal-like deficit in children after cerebellar astrocytoma and medulloblastoma tumor. Behavioral and Brain Functions, 4, 18.

Von Hoff, K., Kieffer, V., Habrand, J.L., Kalifa, C., Dellatolas, G., \& Grill, J. (2008). Impairment of intellectual functions after surgery and posterior fossa irradiation in children with ependymoma is related to age and neurologic complications. BMC Cancer, 8, 15. 